\title{
Tralokinumab: First Approval
}

\author{
Sean Duggan ${ }^{1}$
}

Published online: 18 August 2021

(c) Springer Nature 2021, corrected publication 2021

\begin{abstract}
Tralokinumab (Adtralza ${ }^{\circledR}$ ) is a human IgG4 monoclonal antibody being developed by LEO Pharma for the treatment of atopic dermatitis. The T-helper cytokine IL-13 is thought to play a key role in the pathogenesis of atopic dermatitis. Tralokinumab specifically binds with high affinity to IL-13, inhibiting its interaction with the IL-13 receptor and thereby neutralising the biological activity of the cytokine. Based on results from the ECZTRA 1-3 trials, tralokinumab has recently been approved in the EU for the treatment of moderate-to-severe atopic dermatitis in adult patients who are candidates for systemic therapy. This article summarizes the milestones in the development of tralokinumab leading to this first approval for atopic dermatitis.
\end{abstract}

\section{Digital Features for this AdisInsight Report can be found at https://doi.org/10.6084/m9.figshare.15071697}

\section{Tralokinumab (Adtralza ${ }^{\oplus}$ ): Key points}

A human IgG4 monoclonal antibody is being developed by LEO Pharma for the treatment of atopic dermatitis

Received its first approval on 22 June 2021 in the EU

Approved for the treatment of moderate-to-severe atopic dermatitis in adult patients who are candidates for systemic therapy

\section{Introduction}

Atopic dermatitis is a chronic inflammatory skin disease that is characterized by pruritus and skin barrier dysfunction [1, 2]. While the pathogenesis of atopic dermatitis is multifactorial, recent evidence has identified T-helper (Th) 2 cytokines,

This profile has been extracted and modified from the AdisInsight database. AdisInsight tracks drug development worldwide through the entire development process, from discovery, through preclinical and clinical studies to market launch and beyond.

Sean Duggan

dru@adis.com

1 Springer Nature, Mairangi Bay, Private Bag 65901, Auckland 0754, New Zealand in particular interleukin (IL)-13, as key components of the underlying inflammation leading to the disease $[1,3,4]$. The over-expression of IL-13 cytokine in skin cells results in the down regulation and inhibition of several proteins essential for skin-barrier function, such as filaggrin and loricrin [1]. Recently, IL-13 cytokine has been found to be the dominant cytokine in lesional atopic dermatitis skin and in addition, levels of IL-13 (mRNA and protein) have been shown to correlate with disease severity [4-6]. IL-13 also promotes the recruitment of eosinophils and activated T-cells, which amplifies the Type 2 inflammation [5]. Furthermore, by reducing the keratinocyte antimicrobial peptide production, IL-13 predisposes the skin to Staphylococcus aureus colonization and raises the risk of infection [5].

Tralokinumab is a first in class, fully human IgG4 monoclonal antibody that binds specifically to IL-13 with high affinity, preventing interaction with the IL-13 receptor and subsequent downstream signalling, thereby inhibiting the induction of inflammatory mediators and down-regulation of genes involved in the maintenance of the skin barrier [6].

Tralokinumab was approved on 22 June 2021 in the EU [7] for the treatment of moderate-to-severe atopic dermatitis in adult patients who are candidates for systemic therapy [8]. The drug received a Complete Response Letter in the USA for this indication, with additional data requested relating to a device component of tralokinumab; no additional data on clinical efficacy or safety of the drug product formulation was requested [9]. Tralokinumab was clinically evaluated for the treatment of severe uncontrolled asthma in phase 3 trials [10], although inconsistent beneficial effects on annualized asthma exacerbation rate (primary endpoint) led to development in this indication being discontinued. Clinical 


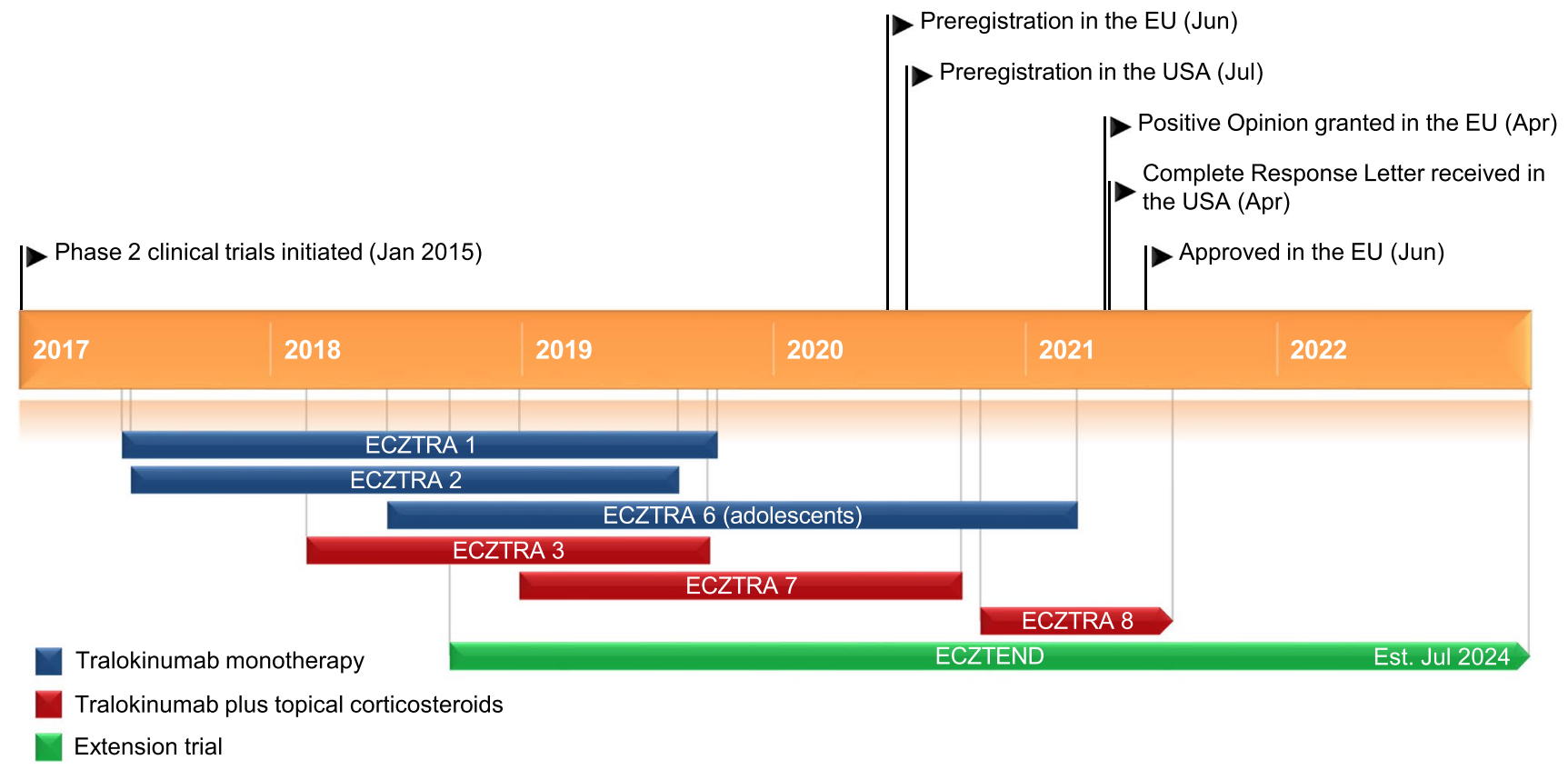

Key milestones in the development of tralokinumab for the treatment of atopic dermatitis, focussing on phase 3 clinical trials.

evaluation of tralokinumab for idiopathic pulmonary fibrosis, COPD and ulcerative colitis has also been discontinued.

The recommended dosage of tralokinumab is an initial dose of $600 \mathrm{mg}$ (four $150 \mathrm{mg}$ subcutaneous injections) followed by $300 \mathrm{mg}$ (two $150 \mathrm{mg}$ subcutaneous injections) administered every other week [8]. A dosage regimen of tralokinumab once every 4 weeks may be considered for patients who achieve clear or almost clear skin after 16 weeks of treatment; the probability of maintaining clear or almost clear skin may be lower with tralokinumab once every 4 weeks [8]. Tralokinumab can be used with or without topical corticosteroids (TCS); topical calcineurin inhibitors may be used, but should be reserved for problem areas only. A patient may self-inject tralokinumab or the patient's caregiver may administer tralokinumab if their healthcare professional determines that this is appropriate. Proper training should be provided to patients and/or caregivers on the administration of tralokinumab prior to use.

\subsection{Company Agreements}

AstraZeneca (MedImmune) granted LEO Pharma an exclusive, global license for tralokinumab in skin diseases in an agreement that was completed in August 2016, with LEO Pharma obtaining exclusive global rights to tralokinumab in atopic dermatitis and any future additional dermatology indications [11, 12]. AstraZeneca will manufacture and supply tralokinumab to LEO Pharma, with AstraZeneca retaining all rights to the drug in any other indications outside of dermatology.

\section{Scientific Summary}

\subsection{Pharmacodynamics}

Tralokinumab, a fully human IgG4 monoclonal antibody, specifically binds to the type 2 cytokine IL-13 with high affinity, thereby inhibiting its interaction with IL-13 receptors, the IL-13R $\alpha 1 / \mathrm{IL}-4 \mathrm{R} \alpha$ receptor complex, and neutralising the biological activity of IL-13 [8]. In an in vitro study in human skin cells, tralokinumab modulated atopic dermatitisassociated genes regulated by IL-13 (e.g. CCL2, CCL26, NTRK1, IL13RA2 in keratinocytes and CCL2, CCL11 and POSTN in dermal fibroblasts), resulting in a dose-dependent and full inhibition of the inflammatory markers at subnanomolar tralokinumab $\mathrm{IC}_{50}$ values [6].

In clinical trials in patients with moderate-to-severe atopic dermatitis, treatment with tralokinumab resulted in a reduction in type 2 signature inflammation biomarkers in both lesional skin (CCL17, CCL18 and CCL26) and in blood (CCL17, periostin and IgE) [8, 13]. Tralokinumab treatment was also associated with lesional skin improvements, including reductions in epidermal thickness and an increase in loricrin (marker of epithelial barrier integrity) $[8,13]$. Tralokinumab treatment was also associated with a $>10$-fold reduction in skin colonization with Staphylococcus aureus $[2,8]$.

The effect of tralokinumab on immune responses to non-live vaccines was assessed in the phase 2 ECZTRA 5 
study (NCT0356237) in adult patients with atopic dermatitis [14]. Subcutaneous tralokinumab administered at an initial dose of $600 \mathrm{mg}$ (four $150 \mathrm{mg}$ injections) followed by $300 \mathrm{mg}$ every second (other) week for 12 weeks had no impact on the immune response to each of component of a combined tetanus, diphtheria and acellular pertussis vaccine, and a meningococcal vaccine, when assessed 4 weeks later $[8,14]$. Therefore, patients receiving tralokinumab may receive concurrent inactivated or non-live vaccinations $[8,14]$.

\subsection{Pharmacokinetics}

Tralokinumab demonstrates linear pharmacokinetics, with exposure of tralokinumab increasing proportionally over the dosages evaluated (150-600 mg) [8]. Subcutaneous tralokinumab has an estimated absolute bioavailability of $76 \%$ [8]. Following single subcutaneous injections of tralokinumab 150 and $300 \mathrm{mg}$ in healthy adult subjects $(n=20)$, the bioavailability was $62 \%$ and $60 \%$, respectively [15].

The median time to $t_{\max }$ was 5-8 days following a subcutaneous dose of tralokinumab [8]. With a 600-mg starting dose followed by tralokinumab $300 \mathrm{mg}$ subcutaneously once every 2 weeks, steady-state pharmacokinetics were reached by week 16 . Across phase III clinical trials, a dosage regimen of tralokinumab $300 \mathrm{mg}$ every 2 weeks produced a mean steady-state trough concentration ranging between 98 and $101 \mu \mathrm{g} / \mathrm{mL}$. Tralokinumab has an estimated total volume of distribution of approximately $4.2 \mathrm{~L}$ [8].
Although the specific pathways have not been determined, as tralokinumab is a protein it is expected to degrade to small peptides and amino acids [8]. Elimination of tralokinumab is via non-saturable proteolytic pathway. Tralokinumab has an elimination halflife of 22 days, which is consistent with the typical estimate for human IgG4 monoclonal antibodies that target soluble cytokines. Across phase III clinical trials, population pharmacokinetic analyses estimated tralokinumab clearance rate to be $0.149 \mathrm{~L} / \mathrm{day}$. In phase 1 trials where tralokinumab was administered intravenously, the estimated clearance rate was 0.179 to $0.211 \mathrm{~L} /$ day [8].

Higher body weight was associated with lower tralokinumab trough concentrations; for patients with high body weight $(>100 \mathrm{~kg}$ ) who achieve clear or almost clear skin after 16 weeks of treatment, reducing the dosage frequency to every fourth week might not be appropriate [8].

Elderly age does not appear to affect tralokinumab pharmacokinetics to a clinically relevant extent; the pharmacokinetic profile of tralokinumab in paediatric patients has not yet been studied. No formal studies have been conducted to investigate any possible impact of hepatic or renal impairment on tralokinumab pharmacokinetics [8]. The effects of tralokinumab on the pharmacokinetics of CYP substrates are being investigated and results are awaited [8].

\section{Features and properties of drug name}

\begin{tabular}{|c|c|}
\hline Alternative names & Adtralza; CAT-354; LP-0162 \\
\hline Class & Anti-inflammatories; Antiasthmatics; Antifibrotics; Monoclonal antibodies; Skin disorder therapies \\
\hline Mechanism of action & Interleukin 13 inhibitors \\
\hline Route of administration & Subcutaneous injection \\
\hline Pharmacodynamics & $\begin{array}{l}\text { Specifically inhibits the interaction of IL-13 with the IL-13R } \alpha 1 / \mathrm{IL}-4 \mathrm{R} \alpha \text { receptor complex, neutralising the } \\
\text { biological activity of IL-13 }\end{array}$ \\
\hline Pharmacokinetics & $\begin{array}{l}\text { Median time to } t_{\max } 5-8 \text { days after a single dose; reaches steady-state pharmacokinetics by } 16 \text { weeks; } \\
\text { estimated absolute bioavailability of } 76 \% \text {; estimated total volume of distribution is } \sim 4.2 \mathrm{~L} \text {; clearance rate } \\
0.149 \mathrm{~L} / \text { day; elimination half-life of } 22 \text { days }\end{array}$ \\
\hline Most common adverse reactions & $\begin{array}{l}\text { Upper respiratory tract infections (mainly reported as common cold), injection site reactions, conjunctivitis, } \\
\text { allergic conjunctivitis }\end{array}$ \\
\hline \multicolumn{2}{|l|}{ ATC codes } \\
\hline WHO ATC code & D11A-H07 (Tralokinumab); D11A-X (Other dermatologicals); R (Respiratory System) \\
\hline EphMRA ATC code & $\begin{array}{l}\text { D11A (Other Dermatological Preparations); D5 (Nonsteroidal Products for Inflammatory Skin Disorders); R } \\
\text { (Respiratory System) }\end{array}$ \\
\hline Chemical name & $\begin{array}{l}\text { Immunoglobulin G4, anti-(human interleukin 13) (human monoclonal CAT-354 heavy chain), disulfide with } \\
\text { human monoclonal CAT-354 light chain, dimer }\end{array}$ \\
\hline
\end{tabular}




\subsection{Therapeutic Trials}

\subsubsection{Monotherapy}

The therapeutic efficacy of tralokinumab monotherapy was evaluated in two identical randomized, double-blind, 52-week, placebo-controlled phase 3 trials in adult patients with moderate-to-severe atopic dermatitis, ECZTRA $1(n=802)$ and ECZTRA $2(n=794)$ [2]. Following a wash-out period of 4 weeks for systemics and 2 weeks for topical treatments, patients were randomized (3:1) to placebo or subcutaneous (SC) tralokinumab $300 \mathrm{mg}$ every 2 weeks for 16 weeks. Patients received $600 \mathrm{mg}$ of tralokinumab or placebo (loading dose) on day 0 . After the 16-week treatment period, patients achieving the primary endpoints of clinical response (Investigator's Global Assessment [IGA] score of 0 [clear] or 1 [almost clear] or 75\% improvement in Eczema Area and Severity Index [EASI 75]) were re-randomized (2:2:1) to placebo or tralokinumab $300 \mathrm{mg}$ every 2 or every 4 weeks, or placebo for 36 weeks; placebo responders at 16 weeks continued receiving placebo to maintain study blinding, although were not included in analyses beyond week 16. Patients treated with tralokinumab or placebo who did not achieve the primary endpoints continued on open -label tralokinumab $300 \mathrm{mg}$ every 2 weeks with optional topical corticosteroids [2].
In ECZTRA 1 and 2, a significantly greater proportion of patients receiving tralokinumab than placebo achieved the primary endpoints at week 16 of IGA score of 0 or 1 $(15.8 \%$ vs $7.1 \%$ in ECZTRA $1[p=0.002]$ and $22.2 \%$ vs $10.9 \%$ in ECZTRA $2[p<0.001])$ and EASI $75(25.0 \%$ vs $12.7 \%$ [ $p<0.001]$ and $33.2 \%$ vs. $11.4 \%$ [ $p<0.001]$ ). Tralokinumab treatment was also associated with significant $(p<0.01)$ improvements in the proportion of patients achieving a reduction in weekly average worst daily pruritus numerical rating scale (NRS) of $\geq 4$ points $(20.0 \%$ vs $10.3 \%$ for placebo in ECZTRA 1; $25.0 \%$ vs $9.5 \%$ in ECZTRA 2), and mean changes in SCORing Atopic Dermatitis (SCORAD) $[-25.2$ vs $-14.7 ;-28.1$ and - 14.0] and Dermatology Life Quality Index (DLQI) $[-7.1$ vs $-5.0 ;-8.8$ vs -4.9$]$ scores [2].

Following 52 weeks of maintenance therapy, the majority of tralokinumab recipients maintained clinical responses achieved at week 16 without the use of rescue medication, including TCS [2]. In a prespecified pooled analysis of patients in ECZTRA 1 and 2 who achieved clinical response with tralokinumab at week 16 , response rates (IGA $0 / 1$ or EASI-75) at week 52 were $56.2 \%$ and $50 \%$ for tralokinumab $300 \mathrm{mg}$ every 2 weeks and tralokinumab $300 \mathrm{mg}$ every 4 weeks, respectively [8]. Of the tralokinumab recipients who did not achieve IGA 0/1 or EASI-75 at week 16 and

Key clinical trials of tralokinumab

\begin{tabular}{|c|c|c|c|c|c|c|}
\hline $\operatorname{Drug}(\mathrm{s})$ & Organisation (s) & Indication & Phase & Status & Location(s) & Identifier \\
\hline Tralokinumab & LEO Pharma & Atopic dermatitis & Phase 3 & Completed & $\begin{array}{l}\text { France, Germany, Japan, } \\
\text { Spain, USA }\end{array}$ & $\begin{array}{c}\text { ECZTRA 1, NCT03131648, } \\
\text { EudraCT2016-004200-65 }\end{array}$ \\
\hline Tralokinumab & LEO Pharma & Atopic dermatitis & Phase 3 & Completed & Multinational & $\begin{array}{r}\text { ECZTRA 2, NCT03160885, } \\
\text { EudraCT2016-004201-13 }\end{array}$ \\
\hline Tralokinumab & LEO Pharma & Atopic dermatitis & Phase 3 & Completed & $\begin{array}{l}\text { Belgium, Canada, England, } \\
\text { Germany, Netherlands, } \\
\text { Poland, Spain, United King- } \\
\text { dom, USA }\end{array}$ & $\begin{array}{c}\text { ECZTRA 3, NCT03363854, } \\
\text { EudraCT2017-002065-21 }\end{array}$ \\
\hline Tralokinumab & LEO Pharma & Atopic dermatitis & Phase 3 & Completed & Multinational & $\begin{array}{r}\text { ECZTRA 6, NCT03526861, } \\
\text { EudraCT2017-005143-33 }\end{array}$ \\
\hline Tralokinumab & LEO Pharma & Atopic dermatitis & Phase 3 & leted & $\begin{array}{l}\text { Belgium, Czech Republic, } \\
\text { England, France, Germany, } \\
\text { Poland, Scotland, Spain, } \\
\text { United Kingdom, Wales }\end{array}$ & $\begin{array}{r}\text { ECZTRA 7, NCT03761537, } \\
\text { EudraCT2018-000747-76 }\end{array}$ \\
\hline Tralokinumab & LEO Pharma & Atopic dermatitis & Phase 3 & Ongoing & Japan & ECZTRA 8, NCT04587453 \\
\hline Tralokinumab & LEO Pharma & Atopic dermatitis & Phase 3 & Ongoing & Multinational & $\begin{array}{r}\text { ECZTEND, NCT03587805, } \\
\text { EudraCT2018-000746-19 }\end{array}$ \\
\hline Tralokinumab & LEO Pharma & Atopic dermatitis & Phase 2 & Completed & Canada, USA & ECZTRA 5, NCT03562377 \\
\hline Tralokinumab & LEO Pharma & Atopic dermatitis & Phase 2 & Ongoing & Germany & $\begin{array}{l}\text { TraSki, NCT04556461, } \\
\text { EudraCT2019-000598-22 }\end{array}$ \\
\hline Tralokinumab & $\begin{array}{l}\text { AstraZeneca, } \\
\text { MedImmune }\end{array}$ & Atopic dermatitis & Phase 2 & Completed & $\begin{array}{l}\text { Australia, Canada, Germany, } \\
\text { Japan, Poland, USA }\end{array}$ & $\begin{array}{l}\text { NCT02347176, } \\
\text { EudraCT2014-003725-17 }\end{array}$ \\
\hline Tralokinumab & LEO Pharma & Atopic dermatitis & hase 1 & Completed & France, Netherlands, USA & $\begin{array}{c}\text { ECZTRA 4, NCT03556592, } \\
\text { EudraCT2018-000534-35 }\end{array}$ \\
\hline
\end{tabular}


were transferred to open-label tralokinumab $300 \mathrm{mg}$ every 2 weeks plus optional TCS, at the week 52 timepoint, $20.8 \%$ in ECZTRA 1 and $19.3 \%$ in ECZTRA 2 achieved IGA 0 or 1, and $46.1 \%$ in ECZTRA 1 and $39.3 \%$ in ECZTRA 2 achieved EASI-75; the clinical response observed was shown to be attributable to tralokinumab treatment rather than optional TCS [8].

\subsubsection{Combination with Topical Corticosteroids}

2.3.2.1 Phase 3 Trials The efficacy and safety of tralokinumab in combination with TCS was evaluated in the doubleblind, randomized, placebo-controlled, phase 3 ECZTRA 3 trial in adults with moderate-to-severe atopic dermatitis who were candidates for systemic therapy $(n=380)$ [16]. Following a wash-out period of 4 weeks for systemics and 2 weeks for topical treatments, patients were randomized to an initial dose of tralokinumab $600 \mathrm{mg}$ (four $150 \mathrm{mg}$ injections) on day 1 , followed by tralokinumab $300 \mathrm{mg}$ every 2 weeks up to week $16(n=253)$ or to matching placebo $(n=127)$. All patients were instructed to apply a thin layer of the supplied TCS as needed, once daily to areas with active lesions [mometasone furoate $0.1 \%$ cream; Europe class 3 (potent); US class 4 (mid-strength), provided free of charge in kit sizes of 180-200 g every 2 weeks]. Patients were instructed to return used and unused tubes at each trial visit to allow measurement of the amount of TCS used. Patients achieving the primary endpoints (IGA 0/1 or EASI 75) at week 16 with initial tralokinumab plus TCS treatment were re-randomized to maintenance treatment with tralokinumab $300 \mathrm{mg}$ every 2 weeks plus TCS or tralokinumab $300 \mathrm{mg}$ every 4 weeks plus TCS for 16 weeks (32 weeks' cumulative use); placebo responders at 16 weeks continued receiving placebo to maintain study blinding, although were not included in analyses beyond 16 weeks. Patients who did not respond to treatment at week 16 (tralokinumab or placebo) continued to receive tralokinumab $300 \mathrm{mg}$ every 2 weeks plus TCS, irrespective of their initial treatment.

In ECZTRA 3, a significantly greater proportion of tralokinumab plus TCS recipients than placebo recipients achieved the primary endpoints of IGA $0 / 1$ (38.9\% vs $26.2 \%$; $p=0.015)$ and EASI $75(56.0 \%$ vs $35.7 \%$; $p<$ $0.001)$ at week 16 [16]. Tralokinumab was also associated with significant improvements in key secondary endpoints compared with placebo; a significantly greater proportion of tralokinumab plus TCS recipients than placebo plus TCS recipients achieved a $\geq 4$-point reduction in weekly average of worst daily pruritus NRS score $(45.4 \%$ vs $34.1 \%$; $p<0.05)$ after 16 weeks, while significant $(p<0.001)$ improvements in SCORAD (- 37.7 vs -26.8$)$ and total DLQI (- 11.7 vs -8.8$)$ scores were also seen with tralokinumab at this timepoint. At week 16, patients receiving tralokinumab $300 \mathrm{mg}$ every 2 weeks had used $50 \%$ less of the supplied TCS compared with patients receiving placebo $[8,16]$. Of the patients who had met the primary endpoints with tralokinumab plus TCS at week $16(n=138)$, the IGA $0 / 1$ and EASI 75 response was maintained at week 32 in high proportion of patients who continued receive tralokinumab every $2(89.6 \%$ and $92.5 \%)$ or $4(77.6 \%$ and $90.8 \%)$ weeks; the maintained response was not associated with an increased use of TCS [16]. In patients receiving tralokinumab every 2 weeks who did not achieve IGA $0 / 1$ and EASI 75 at 16 weeks $(n=95), 30.5 \%$ and $55.8 \%$ achieved these endpoints at week 32 ,indicating that the initial partial response may subsequently improve further with continued treatment every 2 weeks beyond 16 weeks.

2.3.2.2 Phase 2 Trials Tralokinumab was associated with improvements in atopic dermatitis symptoms in a randomized, double-blind, placebo-controlled, doseranging phase $2 \mathrm{~b}$ study in adult patients with moderateto-severe atopic dermatitis receiving concomitant TCS (NCT02347176) [17]. Following a 2-week run in period with TCS, patients $(n=204)$ were randomized 1:1:1:1 to tralokinumab 45,150 or 300 mg every 2 weeks for 12 weeks or placebo. At 12 weeks, mean adjusted improvements from baseline in EASI score (co-primary endpoint) in tralokinumab 45,150 and $300 \mathrm{mg}$ recipients were $-2.89,-4.36$ and -4.94 , respectively, relative to placebo, with statistical significance $(p<0.05)$ reached for the tralokinumab 150 and $300 \mathrm{mg}$ groups. The proportion of tralokinumab 45 , 150 , and $300 \mathrm{mg}$ recipients achieving an IGA response (of 0 or 1 and reduction of $\geq 2$ grades from baseline; co-primary endpoint) was $11.6 \%, 19.5 \%$ and $26.7 \%$ at week 12 , respectively, and was $11.8 \%$ in placebo recipients [17].

\subsection{Adverse Events}

Tralokinumab was generally well tolerated in clinical trials in patients with moderate-to-severe atopic dermatitis $[2,3$, $8,16]$. A pooled safety analysis of the three pivotal phase 3 studies (ECZTRA 1, 2, and 3), the dose-ranging phase 2b study (NCT02347176) and the vaccine-response study (ECZTRA 5) evaluated the tolerability profile of tralokinumab, with or without concomitant TCS $(n=1991)$. During the initial treatment period, the proportion of patients who experienced $\geq 1$ treatment-emergent adverse event was similar between tralokinumab and placebo recipients $(65.7 \%$ vs $67.2 \%$, respectively). Most adverse events were mild or moderate; severe AEs occurred in $4.6 \%$ of the tralokinumab group and $6.3 \%$ of the placebo group. The proportion of patients who discontinued treatment due to adverse events was low (2.3\% and $2.8 \%$ in tralokinumab and placebo recipients, respectively).

The most common adverse events reported during the initial 16-week treatment period were upper respiratory tract 
infections (mainly reported as common cold), injection site reactions, conjunctivitis and allergic conjunctivitis [8, 18]. Conjunctivitis occurred in $5.4 \%$ of tralokinumab recipients and $1.9 \%$ of placebo recipients; most patients recovered or were recovering during the treatment period. Eosinophilia occurred in $1.3 \%$ of tralokinumab recipients (vs $0.3 \%$ for placebo) during the initial 16-week treatment period, although increases from baseline observed were transient and mean eosinophil counts returned to baseline with continued treatment [8]. The rate of injection site reactions was higher with tralokinumab treatment than with placebo during the initial 16 -week treatment period ( $7.2 \%$ vs $3.0 \%)$; however, the vast majority of events were of mild to moderate severity and few required treatment discontinuation; most (76\%) injection site reactions resolved within 1 to 5 days. Tralokinumab treatment was associated with a lower rate of skin infections requiring systemic treatment ( $2.5 \%$ vs $5.5 \%$ for placebo) and eczema herpeticum $(0.3 \%$ vs $1.5 \%)$ compared with placebo in the initial treatment period $[8,18]$.

As with all therapeutic proteins, tralokinumab has a potential for immunogenicity. In clinical trials, anti-drug antibodies were detected in $1.4 \%$ tralokinumab recipients compared with $1.3 \%$ for placebo, and neutralising antibodies seen in $0.1 \%$ of tralokinumab recipients and $0.2 \%$ of placebo recipients. [8]. The presence of anti-drug antibodies was not associated with any impact on exposure, efficacy and safety of tralokinumab. No immunogenicity related adverse events (such as immune complex disease, serum sickness/serum sickness-like reactions, or anaphylaxis) were observed with tralokinumab in patients with atopic dermatitis [3].

The long-term tolerability profile of tralokinumab up to 52 weeks (as monotherapy in ECZTRA 1 and 2 [2]) and up to 32 weeks (plus TCS in ECZTRA 3 [16]) was consistent with that observed over the initial 16-week treatment period [8].

\subsection{Ongoing Clinical Trials}

Phase III clinical trials evaluating tralokinumab in patients with atopic dermatitis are ongoing, namely the ECZTRA 8 trial (NCT04587453) in Japanese patients with moderateto-severe atopic dermatitis $(n=126)$ and the long-term (5 year) safety and efficacy extension trial ECZTEND (NCT03587805) in patients with atopic dermatitis who participated in previous tralokinumab trials $(n=1600)$. Interim analyses from ECZTEND have shown tralokinumab to be generally well tolerated with 1235.7 patient-years of exposure and consistent with parent trials $(n=1174)$ [19], and efficacy was maintained through 56 weeks of treatment (49.7\% achieved IGA $0 / 1$ and $82.8 \%$ achieved EASI 75 , compared with parent trial baseline) [20]. An open label, phase II clinical trial TraSki (NCT04556461) investigating the effects of tralokinumab on skin barrier physiology and function in patients with atopic dermatitis $(n=16)$ is also ongoing in Germany.

In addition, a number of trials have recently completed and are awaiting results: the phase 3 trial ECZTRA 6 (NCT03526861) evaluating tralokinumab in monotherapy in adolescents with moderate-to-severe atopic dermatitis; the phase 3 ECZTRA 7 (NCT03761537) evaluating tralokinumab plus TCS in patients with severe atopic dermatitis who have contraindications or disease that is not well controlled with oral cyclosporine; and the phase 1 trial ECZTRA 4 (NCT03556592) evaluating drug interactions with tralokinumab.

\section{Current Status}

Tralokinumab received its first approval on 22 June 2021 for the treatment of moderate-to-severe atopic dermatitis in adult patients who are candidates for systemic therapy in the EU.

Supplementary Information The online version contains supplementary material available at https://doi.org/10.1007/s40265-021-01583-1.

Funding The preparation of this review was not supported by any external funding.

\section{Declarations}

Authorship and Conflict of interest During the peer review process the manufacturer of the agent under review was offered an opportunity to comment on the article. Changes resulting from any comments received were made by the authors on the basis of scientific completeness and accuracy. Sean Duggan is a salaried employee of Adis International $\mathrm{Ltd} /$ Springer Nature, and declares no relevant conflicts of interest. All authors contributed to the review and are responsible for the article content.

Ethics approval, Consent to participate, Consent to publish, Availability of data and material, Code availability Not applicable.

Open Access This article is licensed under a Creative Commons Attribution-NonCommercial 4.0 International License, which permits any non-commercial use, sharing, adaptation, distribution and reproduction in any medium or format, as long as you give appropriate credit to the original author(s) and the source, provide a link to the Creative Commons licence, and indicate if changes were made. The images or other third party material in this article are included in the article's Creative Commons licence, unless indicated otherwise in a credit line to the material. If material is not included in the article's Creative Commons licence and your intended use is not permitted by statutory regulation or exceeds the permitted use, you will need to obtain permission directly from the copyright holder. To view a copy of this licence, visit http://creativecommons.org/licenses/by-nc/4.0/. 


\section{References}

1. Newsom M, Bashyam AM, Balogh EA, et al. New and emerging systemic treatments for atopic dermatitis. Drugs. 2020;80(11):1041-52.

2. Wollenberg A, Blauvelt A, Guttman-Yassky E, et al. Tralokinumab for moderate-to-severe atopic dermatitis: results from two 52-week, randomized, double-blind, multicentre, placebocontrolled phase III trials (ECZTRA 1 and ECZTRA 2). Br J Dermatol. 2021;184(3):437-49.

3. European Medicines Agency. Adtralza (tralokinumab): assessment report. 2020. https://www.ema.europa.eu/en/documents/asses sment-report/adtralza-h-c-5255-0000-epar-assessment-report_ en.pdf. Accessed 26 Jul 2021.

4. Tsoi LC, Rodriguez E, Degenhardt F, et al. Atopic dermatitis is an IL-13-dominant disease with greater molecular heterogeneity compared to psoriasis. J Invest Dermatol. 2019;139(7):1480-9.

5. Bieber T. Interleukin-13: targeting an underestimated cytokine in atopic dermatitis. Allergy. 2020;75(1):54-62.

6. Tollenaere MAX, Litman T, Moebus L, et al. Skin barrier and inflammation genes associated with atopic dermatitis are regulated by interleukin-13 and modulated by tralokinumab in vitro. Acta Derm Venereol. 2021. https://doi.org/10.2340/00015555-3810.

7. Leo Pharma A/S. LEO Pharma announces European Commission approval of Adtralza ${ }^{\circledR}$ (tralokinumab) as the first and only treatment specifically targeting IL-13 for adults with moderateto-severe atopic dermatitis [media release]. 22 Jun 2021. https:// www.leo-pharma.com/.

8. European Medicines Agency. Summary of product characteristics: adtralza $150 \mathrm{mg}$ solution for injection in pre-filled syringe. 2021. https://ec.europa.eu/health/documents/community-register/2021/ 20210617151835/anx_151835_en.pdf. Accessed 28 Jun 2021.

9. National Eczema Association. Update on U.S. FDA review of LEO Pharma's biologics license application for tralokinumab for the treatment of adults with moderate-to-severe atopic dermatitis [media release]. 29 Apr 2021. https://nationaleczema.org/ leo-42921/.

10. Panettieri RA Jr, Sjöbring U, Péterffy A, et al. Tralokinumab for severe, uncontrolled asthma (STRATOS 1 and STRATOS 2): two randomised, double-blind, placebo-controlled, phase 3 clinical trials. Lancet Respir Med. 2018;6(7):511-25.

11. AstraZeneca. AZ enters licensing agreements with LEO Pharma [media release]. $1 \mathrm{Jul}$ 2016. http://www.astrazeneca.com.
12. AstraZeneca. AstraZeneca completes agreement with LEO Pharma [media release]. 16 Aug 2016. http://www.astrazeneca. com.

13. Guttman-Yassky E, Zirwas M, Kabashima K ea. Neutralizing interleukin-13 with tralokinumab shifts the molecular phenotype of lesional skin towards that of non-lesional skin and restores skin barrier abnormalities [abstract no. 29421]. In: American Academy of Dermatology Association Virtual Meeting Experience (AAD VMX). 2021.

14. Merola JF, Bagel J, Almgren P, et al. Tralokinumab does not impact vaccine-induced immune responses: results from a 30-week, randomized, placebo-controlled trial in adults with moderate-to-severe atopic dermatitis. J Am Acad Dermatol. 2021. https://doi.org/10.1016/j.jaad.2021.03.032.

15. Oh CK, Faggioni R, Jin F, et al. An open-label, single-dose bioavailability study of the pharmacokinetics of CAT-354 after subcutaneous and intravenous administration in healthy males. Br J Clin Pharmacol. 2010;69(6):645-55.

16. Silverberg JI, Toth D, Bieber T, et al. Tralokinumab plus topical corticosteroids for the treatment of moderate-to-severe atopic dermatitis: results from the double-blind, randomized, multicentre, placebo-controlled phase III ECZTRA 3 trial. Br J Dermatol. 2021;184(3):450-63.

17. Wollenberg A, Howell MD, Guttman-Yassky E, et al. Treatment of atopic dermatitis with tralokinumab, an anti-IL-13 mAb. J Allergy Clin Immunol. 2019;143(1):135-41.

18. Simpson E, Merola JF, Silverberg J, et al. Safety of specifically targeting interleukin 13 with tralokinumab in adult patients with moderate-to-severe atopic dermatitis: pooled analysis of five randomised, double-blind, placebo-controlled Phase 3 and Phase 2 trials [abstract no. P0218 plus poster]. In: 29th Congress EADV virtual-new frontiers in dermatology and venereology. 2020.

19. Blauvelt a, Lacour JP, Toth D, et al. Long-term safety, efficacy, and adherence to tralokinumab treatment in moderate-to-severe atopic dermatitis for up to 3 years: interim readout of ECZTEND, a phase 3, long-term extension trial [abstract no. 27697 plus poster]. In: American Academy of Dermatology Association Virtual Meeting Experience. 2021.

20. Blauvelt A, Lacour JP, Toth D, et al. Long-term improvements observed in tralokinumab-treated patients with moderate-to-severe atopic dermatitis: an ECZTEND interim analysis [abstract no. 29393]. In: American Academy of Dermatology Association Virtual Meeting Experience. 2021. 